\title{
REVIEW \\ Heparin for venous thromboembolism prophylaxis in patients with acute spinal cord injury: a systematic review and meta-analysis
}

\author{
Hong-Lin Chen ${ }^{1}$ and Xiao-Dong Wang ${ }^{2}$
}

\begin{abstract}
Objective: The objective of this study is to systematically review and estimate the effect of heparin for thromboprophylaxis in patients with acute spinal cord injury (SCl).

Methods: We searched the PubMed database up to February 2013. Only randomized control trials (RCTs), quasi-RCTs, cohorts, casecontrol and cross-sectional studies were included. The incidence of venous thromboembolism (VTE) and major bleeding complication were recorded as the endpoints. The summary relative risks (RR) were calculated by meta-analysis.

Results: A total of 18 studies with 2578 patients were included. Four studies evaluated the effects of low-dose unfractionated heparin (LDUH) compared placebo or untreated. No significant differences were observed, with the summary RR 0.661 (95\% confidence interval (CI) 0.365-1.199; $Z=1.36, P=0.173$ ) for VTE. Only one RCT compared fixed-dose LDUH with adjusted-dose LDUH, which showed lower VTE incidence but higher bleeding incidence for adjusted dose. Nine trials have compared LDUH with lowmolecular-weight heparin (LMWH). No significant differences were observed for VTE with the summary RR $1.633(95 \% \mathrm{Cl} 0.822-$ 3.243; $Z=1.40, P=0.162$ ). But major bleeding was lower with $\mathrm{LMWH}$ (summary $\mathrm{RR}=2.034,95 \% \mathrm{Cl} 1.018-4.063 ; Z=2.01$, $P=0.044)$. Three studies compared different LMWHs, which included one for enoxaparin versus tinzaparin and two for enoxaparin versus dalteparin. No significant differences were observed with the summary RR 0.694 (95\% $\mathrm{Cl} 0.336-1.434 ; Z=0.99, P=0.324$ ) for VTE. Three studies compared different dose of LMWH. No differences were observed.

Conclusion: Our meta-analysis showed that in patients with acute SCI, LDUH have no thromboprophylaxis effect compared with placebo or untreated; LMWH seems only can reduce bleeding incidence, but not prophylaxis thromboembolism compared with LDUH. Because of no good quality studies existed in this setting, well-designed RCTs are urgently needed.
\end{abstract}

Spinal Cord (2013) 51, 596-602; doi:10.1038/sc.2013.48; published online 21 May 2013

Keywords: spinal cord injury; venous thromboembolism; heparin; low-dose unfractionated heparin; low-molecular-weight heparin

\section{INTRODUCTION}

Venous thromboembolism (VTE) is one of the major complications after acute spinal cord injury (SCI). VTE includes deep-vein thrombosis (DVT) and pulmonary embolism (PE). The overall incidence of DVT in the patients with SCI ranges from 49 to $100 \%$ in the first 12 weeks, with the first 2 weeks having the highest rate following acute injury. ${ }^{1}$ DVT leads to venous occlusion, edema and post-thrombotic syndrome. Most importantly, when a part of the DVT breaks off and travels through the bloodstream to the lung, it causes PE. PE is the third most common cause of death in patients with SCI. ${ }^{2}$ The incidence of clinical PE ranges from 4 to $10 \%$ and fatal PE ranges from 0.2 to $5 \% .{ }^{3}$ VTE after SCI is associated with high morbidity and high mortality, and may generate significant additional health-related costs. It is important for high-risk patients with SCI to receive VTE prophylaxis.

Currently, mechanical method, pharmacological method and surgical method were used in VTE prophylaxes. ${ }^{4}$ Mechanical methods include intermittent pneumatic compression, pressuregraded elastic stockings, venous foot pump and neuromuscular electrical stimulation. Surgical method is inferior vena cava filters implantation which used to prophylaxis PE. However, pharmacological agents are most widely used. Pharmacological methods include oral anticoagulants, low-dose unfractionated heparin (LDUH) and low-molecular-weight heparin (LMWH). Oral anticoagulants, such as aspirin, dipyridamole and warfarin, did not prove to give satisfactory results. ${ }^{5} \mathrm{LDUH}$ (5000 units, twice daily) was first used in 1975 for VTE prophylaxes in patients with SCI and was recommended by the Consortium for Spinal Cord Medicine Clinical Practice guidelines. ${ }^{6}$ However, some recent study showed LDUH did not provide VTE prophylaxis effect in patients with SCI. ${ }^{7}$ Since 1995, there has been increasing use of LMWH. On the basis of some evidence, LMWH was recommended by the Consortium for Spinal Cord Medicine Clinical Practice ${ }^{6}$ and the American College of Chest Physicians. ${ }^{8}$ Although some recent trials did not prove the affect of VTE prophylaxis in patients with SCI. ${ }^{9,10}$ The results were still controversial in heparin for VTE prophylaxis in patients with acute SCI.

The aim of this study was to systematically review and estimate the effect of heparin for thromboprophylaxis in patients with acute SCI. We want to assess thromboprophylaxis effect by comparing: (1) LDUH versus placebo or untreated; (2) fixed-dose LDUH versus 
adjusted-dose LDUH; (3) LDUH versus LMWH; (4) different kinds of LMWHs (enoxaparin, dalteparin and tinzaparin) and (5) different dose of LMWHs.

\section{MATERIALS AND METHODS}

\section{Search strategy}

A systematic literature review of the MEDLINE database up to February 2013 was initially conducted. The search detail was 'spinal cord injuries' (MeSH Terms) and (thrombosis (Text Word) or 'pulmonary embolism' (Text Word) or thromboembolism (Text Word)) and (heparin (Text Word) or enoxaparin (Text Word) or dalteparin (Text Word) or tinzaparin (Text Word)). We supplemented our searches by manually reviewing the references of all relevant studies.

\section{Eligibility criteria}

The following inclusion criteria had to be fulfilled: (1) types of participants: studies investigated patients with acute SCI receiving heparin for thromboprophylaxis were included; (2) types of studies: randomized control trials (RCT), quasi-RCTs, cohorts, case-control and cross-sectional studies were included; (3) types of intervention: studies comparing different kinds of heparin (LDUH versus placebo or fixed-dose LDUH versus adjusted-dose LDUH, or LMWH versus LDUH, or different kinds of LMWH, or different dose of LMWHs) were included; and (4) types of outcomes: studies that assessed the incidence of VTE or the incidence of major bleeding complication were included. We excluded case reports, case series and reviews. We also excluded the studies that did not provide data to calculate the relative risk (RR) of two outcomes.

\section{Quality assessment}

The quality of included RCTs was assessed using Jadad scale. ${ }^{11}$ The scale incorporates the following five questions: (1) Is the study randomized? (2) Is the study double blinded? (3) Is there a description of withdrawals? (4) Is the randomization adequately described? and (5) Is the blindness adequately described? The quality of included quasi-RCTs, cohorts, case-control and cross-sectional studies was assessed according to the Newcastle-Ottawa Scale (NOS). ${ }^{12}$ The NOS contains eight items and are categorized into three dimensions, including selection (4), comparability (1) and exposure (3). A high-quality study can be awarded a maximum of one star for each numbered item within the selection and exposure categories. A maximum of two stars can be given for comparability. The NOS ranges between zero up to nine stars. The level of evidence of each article was scored using the Oxford Centre for Evidence-based Medicine Level of Evidence scale ${ }^{13}$ Two reviewers independently assessed the quality. Disagreements were resolved by discussion and by seeking the opinion of a third reviewer.

\section{Data extraction}

The following data were abstracted onto standardized forms: first author, publication year, country, study design, the usage of heparin, age of patients, gender of patients, American Spinal Injury Association grade, the incidence DVT, the incidence of PE; the incidence of combined VTE (DVT and PE) and the incidence of major bleeding complication. Data extraction was carried out independently by two reviewers. Disagreements were resolved by discussion between the two.

\section{Statistical analysis}

The meta-analyses was performed with Stata software, version 12.0 (Stata Corp, College Station, TX, USA). Overall effects were determined using the $Z$-test. Statistical heterogeneity was explored by $\chi^{2}$ and inconsistency $\left(I^{2}\right)$ statistics. In the absence of significant heterogeneity, studies were pooled using a fixed-effect model. If heterogeneity was observed, a random-effect model was used. Publication bias was estimated by the funnel plot, the Egger regression test and Begg's-adjusted rank correlation test. Subgroup analyses were carried out by different heparin.

\section{RESULTS}

\section{Characteristics of the included studies}

We identified 18 studies $^{7,9,10,14-29}$ with 2578 patients that met our inclusion criteria for meta-analysis. The detailed steps of our literature search are shown in Figure 1. The studies were conducted in USA, India, Canada, UK and Germany. Of the 18 selected studies, 7 studies were RCTs, 1 was quasi-RCT and the remaining 10 used observational study method (cohorts, case-control and cross-sectional studies). Four of the 18 studies have compared LDUH (dose range: $5000 \mathrm{U}$ twice daily or $5000 \mathrm{U}$ three times daily) with placebo or untreated, one RCT compared fixed-dose LDUH with adjusted-dose LDUH (adjusted to prolong the activated partial thromboplastin time (APTT) to 1.5 times), nine trials have compared LDUH with LMWH (dose range: enoxaparin, $20 \mathrm{mg}$ daily to $30 \mathrm{mg}$ twice daily; tinzaparin, 3500-4500 IU once daily; dalteparin was 5000 IU daily), three studies compared different LMWHs (one for enoxaparin versus tinzaparin, two for enoxaparin versus dalteparin) and the other three studies compared different dose of LMWHs. The Jadad scale for included RCTs scored 2-4 (total 5) and the NOS of the nonrandomised studies scored 5-7 (total 9). Overall, the quality of the studies was not good and low scores were achieved. The study provided level 1b-3b evidence on the Oxford Level of Evidence scale. Table 1 shows the characteristics of the 18 identified studies.

\section{LDUH versus placebo or untreated}

Two RCTs ${ }^{7,29}$ and two case-control studies ${ }^{24,28}$ evaluated the effects of LDUH compared placebo or untreated. The incidence of combined VTE was $1.8-50.0 \%$ in LDUH group, and $3.1-47.1 \%$ in placebo or untreated group. There was no substantial heterogeneity between four studies $\left(\chi_{(3)}^{2}=4.03, P=0.259, I^{2}=25.5 \%\right)$. The summary RR was 0.661 (95\% confidence interval (CI) $0.365-1.199 ; Z=1.36, P=0.173$ ) (Figure 2, the forest plot). The Begg's test $(Z=0.34, P=0.734)$ and the Egger's test $(t=1.15, P=0.369)$ indicated no evidence of publication bias (Figure 2. the funnel plot). Not available data was found to evaluate the major bleeding complication in these four studies.

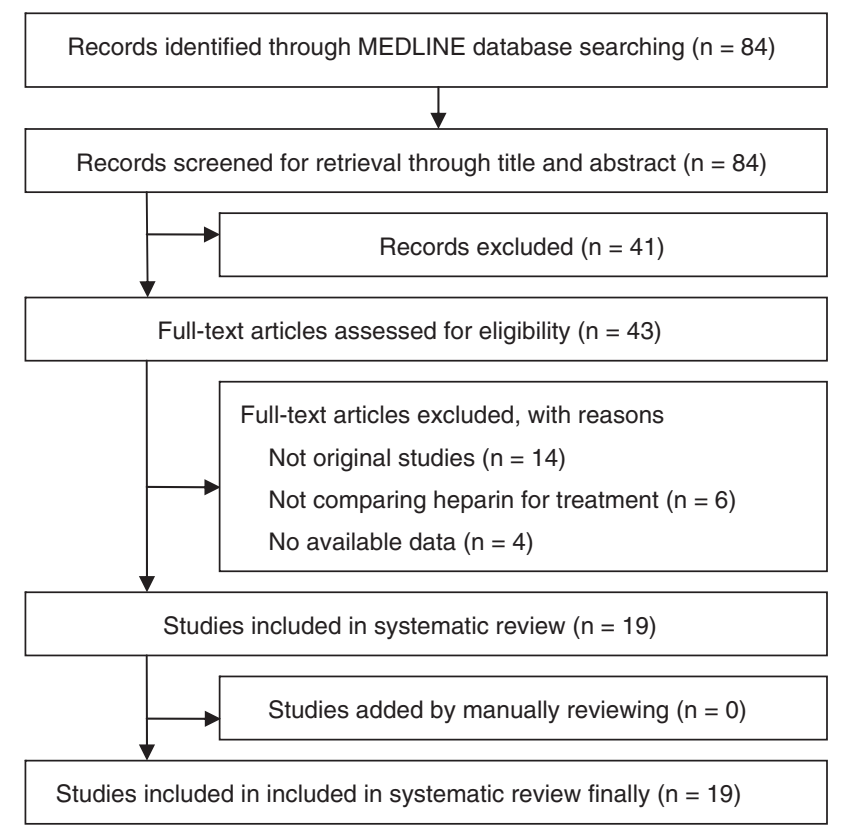

Figure1 Flow diagram showing selection of studies. 


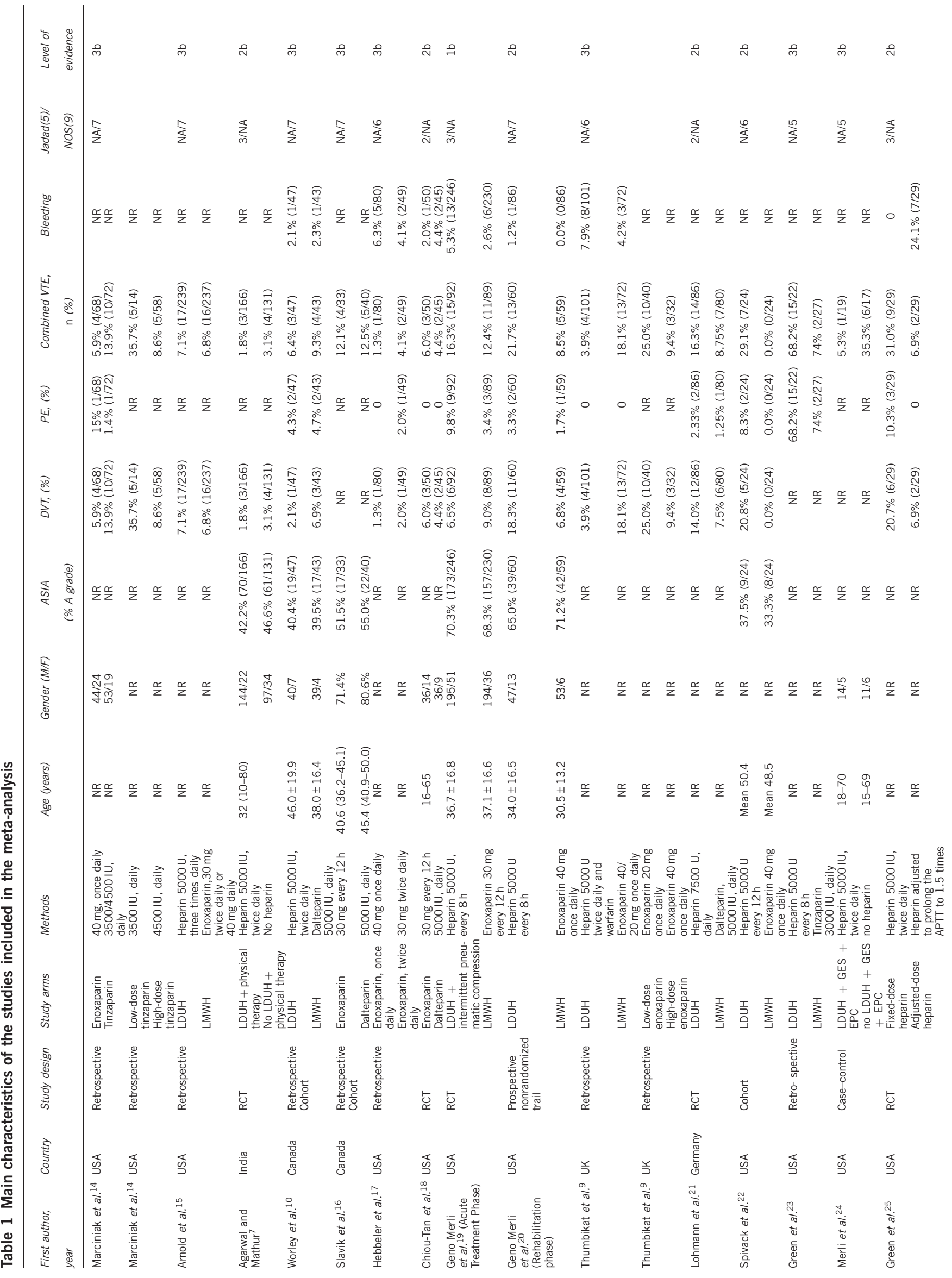




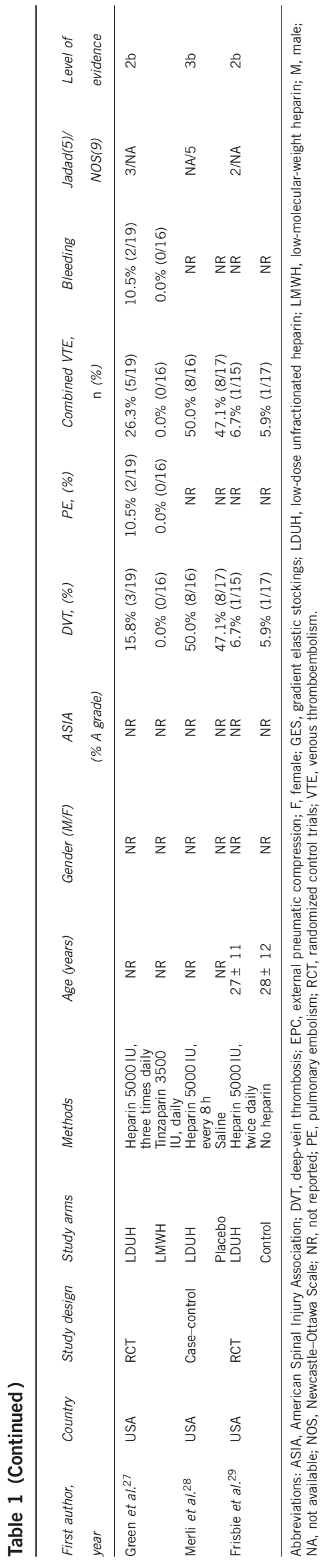

Fixed-dose LDUH versus adjusted-dose LDUH

Only one RCT ${ }^{25,26}$ (same data in two artiles) compared fixed-dose unfractionated heparin with adjusted-dose heparin with level $2 \mathrm{~b}$ evidence. There was no sufficient data for meta-analysis. The incidence of thromboembolism was 31\% (9/29) in the fixed-dose group and 7\% (2/29) in the adjusted-dose group. The RR was 1.923 (95\% CI $\left.1.246-2.966 ; \chi^{2}=5.50, P=0.019\right)$. No patient $(0.0 \%, 0 / 29)$ in the fixed-dose group bled, whereas bleeding occurred in seven patients $(24.1 \%, 7 / 29)$ in the adjusted-dose group $\left(\chi^{2}=7.96\right.$, $P=0.0048)$.

\section{LDUH versus LMWH}

Nine trials , $^{9,15,19-23,27}$ have compared LDUH with LMWH, which included three RCTs, one quasi-RCT, one cohort and four casecontrol studies. Among LMWHs, enoxaparin was the compound most frequently used.

Nine trials ${ }^{9,10,15,19-23,27}$ provided the incidence of VTE between groups. The incidence was $4.0-68.2 \%$ in LDUH group and $0.0-18.1 \%$ in LMWH group. The heterogeneity was found between nine studies $\left(\chi^{2}{ }_{(8)}=27.01, P=0.001, I^{2}=70.4 \%\right)$. The summary RR was 1.633 (95\% CI 0.822-3.243; $Z=1.40, P=0.162$ ) (Figure 3, the forest plot). The Begg's test $(Z=1.77, P=0.076)$ and the Egger's test $(t=2.84$, $P=0.025)$ indicated there was publication bias in the studies (Figure 2. the funnel plot). Although in the heparin versus enoxaparin subgroup, ${ }^{9,15,19,20,22}$ the summary RR was 1.186 (95\% CI 0.502-2.800; $Z=0.39, P=0.697$ ) (Figure 3, top of the forest plot); in the heparin versus dalteparin subgroup, ${ }^{10,21}$ the summary RR was 1.346 (95\% CI 0.539-3.361; $Z=0.64, P=0.525$ ) (Figure 3, middle of the forest plot) in the heparin versus tinzaparin subgroup, ${ }^{23,27}$ the summary RR was 9.232 (95\% CI 2.704-31.519; $Z=3.55, P=0.000$ ) (Figure 3, bottom of the forest plot).

Only five trials ${ }^{9,10,19,20,27}$ provided the incidence of major bleeding complication between groups. The incidence was $1.2-10.5 \%$ in LDUH group, and $0.0-4.2 \%$ in LMWH group. There was no substantial heterogeneity between five studies $\left(\chi_{(4)}^{2}=0.63, P=0.960, I^{2}=0.0 \%\right)$. The summary RR was $2.034(95 \%$ CI $1.018-4.063 ; Z=2.01$, $P=0.044)$ (Figure 4 , the forest plot). The Begg's test $(Z=0.24$, $P=0.806)$ and the Egger's test $(t=0.77, P=0.498)$ indicated no evidence of publication bias (Figure 4. the funnel plot). No available data was found to conduct subgroup analyses.

\section{Different kinds of LMWHs}

Three studies compared different LMWHs (one ${ }^{14}$ for enoxaparin versus tinzaparin, two ${ }^{16,18}$ for enoxaparin versus dalteparin). The incidence was $5.9-12.1 \%$ in enoxaparin group, $4.4-12.5 \%$ in tinzaparin group and $13.9 \%$ in dalteparin group. There was no substantial heterogeneity between three studies $\left(\chi^{2}(2)=1.60\right.$, $\left.P=0.449, I^{2}=0.0 \%\right)$. The summary RR was 0.694 (95\% CI $0.336-$ 1.434; $Z=0.99, P=0.324$ ) (Figure 5, the forest plot). The Begg's test $(Z=1.04, P=0.296)$ and the Egger's test $(t=1.77, P=0.327)$ indicated no evidence of publication bias (Figure 2 the funnel plot). Although in the enoxaparin versus tinzaparin subgroup, the RR was 0.424 (95\% CI 0.139-1.287; $Z=1.52, P=0.130$ ) (Figure 5, top of the forest plot); in the heparin versus dalteparin subgroup, the summary RR was 1.091 (95\% CI $0.400-2.975 ; Z=0.17, P=0.866$ ) (Figure 5, bottom of the forest plot). Only one trial provided the incidence of major bleeding complication between groups. The incidence was $2.0 \%(1 / 50)$ in enoxaparin group and $4.4 \%(2 / 45)$ in dalteparin group. The RR was 0.626 (95\% CI $0.125-3.136 ; \chi^{2}=0.46, P=0.496$ ). 

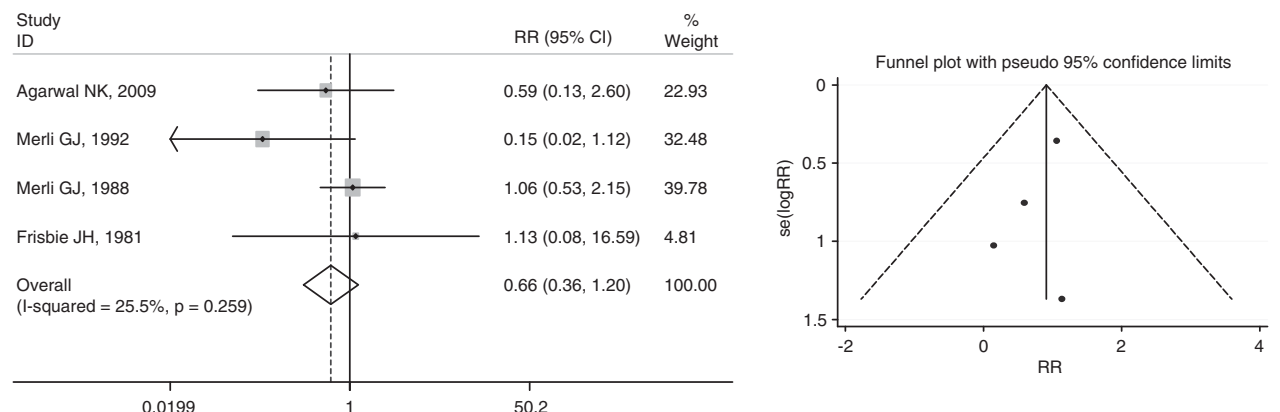

Figure2 VTE incidence in LDUH versus placebo or untreated for patients with SCl. The forest plot (left) showed no significant differences between two groups. The funnel plot (right) showed no evidence of publication bias.

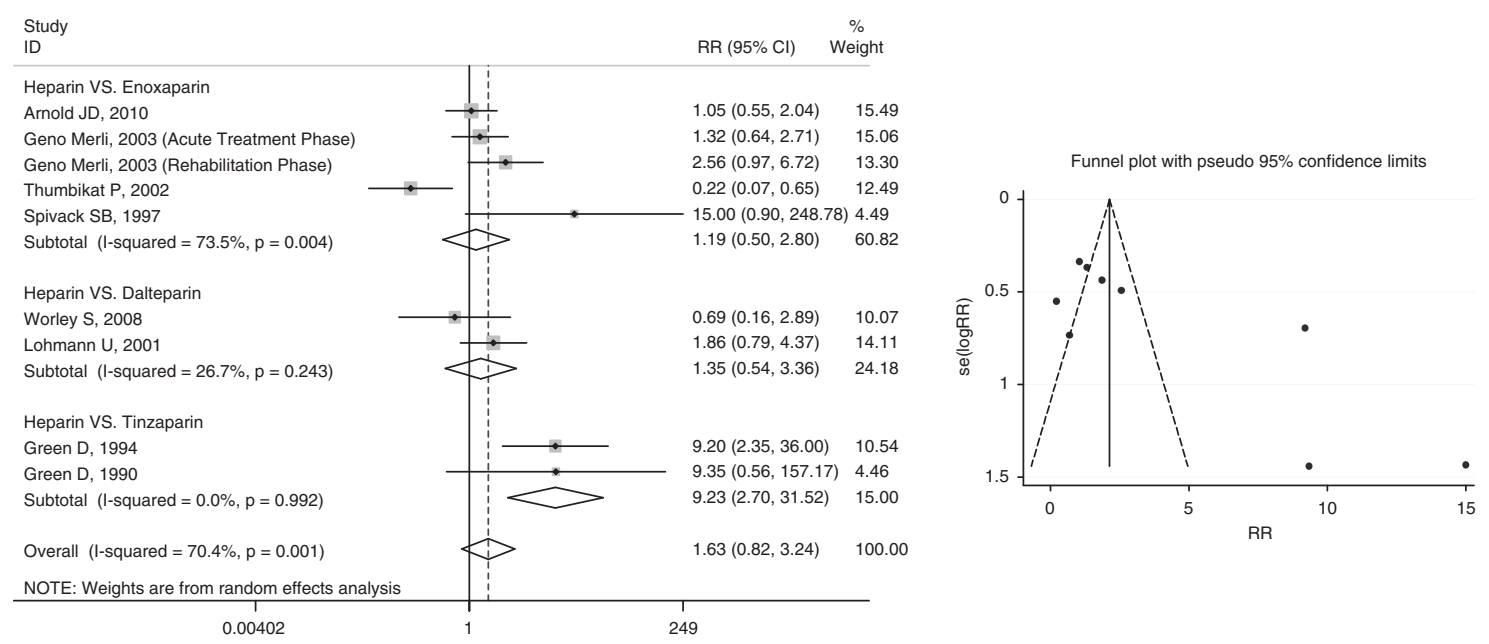

Figure3 VTE incidence in LDUH versus LMWH for patients with SCI. The forest plot (left) showed no significant differences between two groups. In the heparin versus enoxaparin subgroup (top), no significant differences were found; in the heparin versus dalteparin subgroup (middle), no significant differences were found; in the heparin versus tinzaparin subgroup (bottom), tinzaparin showed that it has effect of lower VTE incidence. The funnel plot (right) showed that there was publication bias in the studies.
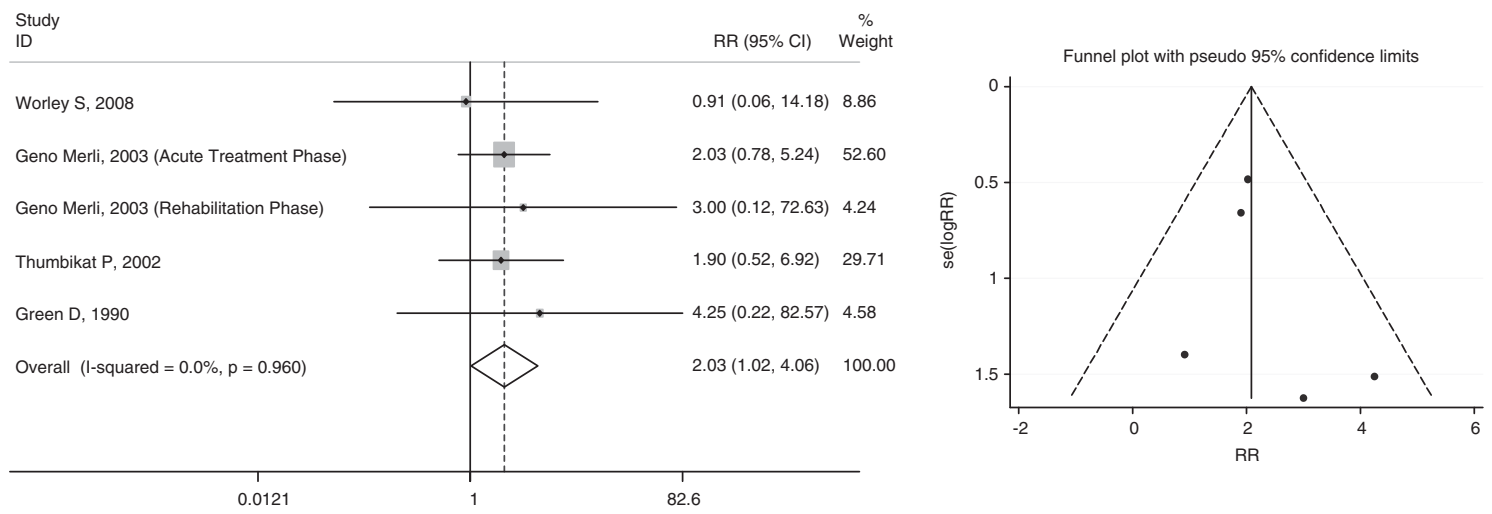

Figure4 Major bleeding complications in LDUH versus LMWH for patients with SCl. The forest plot (left) showed that LMWH has effect of lower bleeding incidence. The funnel plot (right) showed no evidence of publication bias.

\section{Different dose of LMWHs}

One trial ${ }^{14}$ compared 3500 IU tinzaparin daily versus 4500 IU daily. The incidence of thromboembolism was $35.7 \%(5 / 14)$ and $8.6 \%(5 /$ 58), respectively. The RR was 4.143 (95\% CI 1.388-12.366). One trial ${ }^{17}$ compared $40 \mathrm{mg}$ enoxaparin daily versus $30 \mathrm{mg}$ twice daily. The incidence of thromboembolism was $1.3 \%(1 / 80)$ and $4.1 \%(2 / 49)$, respectively. The RR was 0.306 (95\% CI $0.029-3.289)$. One trial ${ }^{9}$ compared $20 \mathrm{mg}$ enoxaparin daily versus $40 \mathrm{mg}$ daily. The incidence of thromboembolism was $25.0 \%(10 / 40)$ and $9.4 \%(3 / 32)$, respectively. The RR was 2.667 (95\% CI 0.800-8.885).

\section{DISCUSSION}

Heparin is a naturally occurring anticoagulant produced by basophils and mast cells. Heparin binds to the enzyme inhibitor antithrombin 


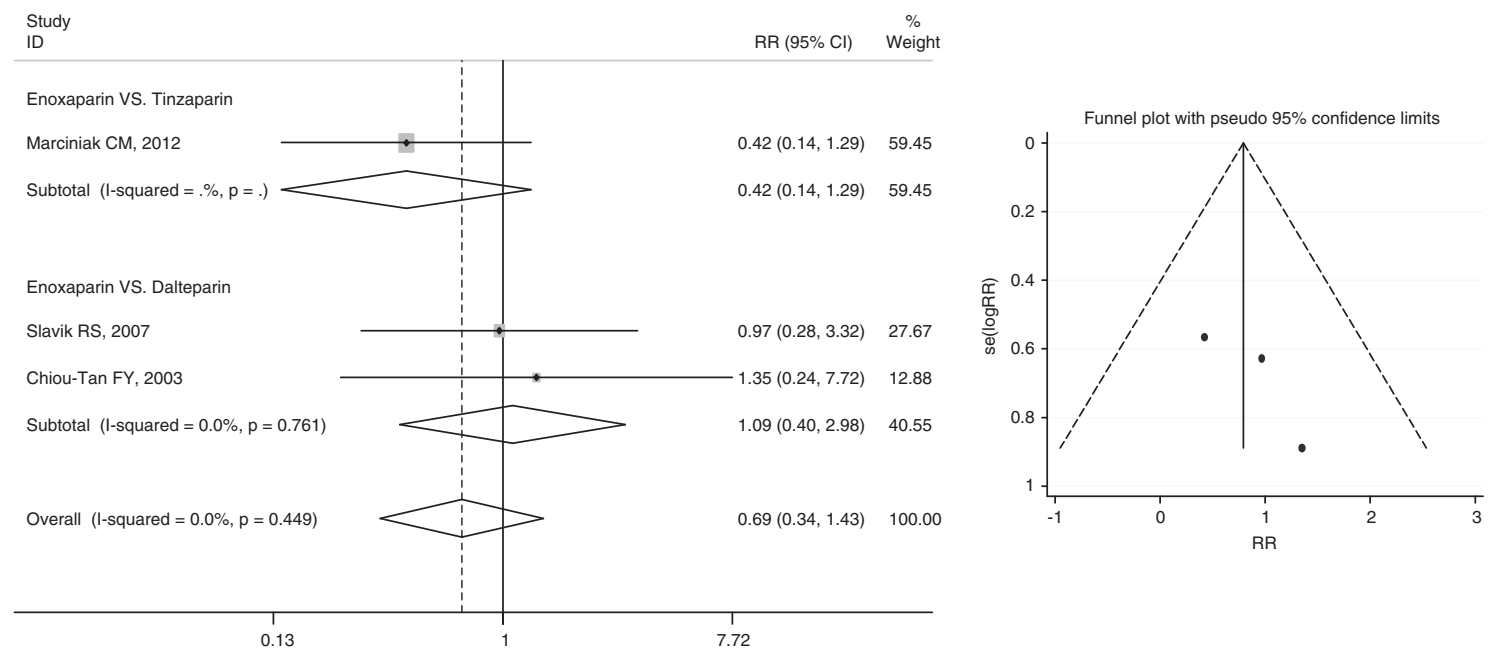

Figure5 VTE incidence in different kinds of LMWH for patients with SCI. The forest plot (left) showed no significant differences between two groups. In the enoxaparin versus tinzaparin subgroup (top), no significant differences were found; in the enoxaparin versus dalteparin subgroup (bottom), no significant differences were found. The funnel plot (right) showed that there was publication bias in the studies.

(AT) III and activates the AT. The activated AT then inactivates thrombin and other proteases involved in blood clotting: XIIa, XIa, IXa and Xa. Heparin acts as an anticoagulant for preventing the formation of clots, blocking extension of existing clots within the blood and never breaking down clots that have already formed. It is generally used for anticoagulation for DVT and PE. Although in our meta-analysis, when LDUH compared with placebo or untreated in patients with acute SCI, the summary RR for VTE was 0.661 , with 95\% CI 0.365-1.199. Although only four trails, with two RCTs and two case-control studies, were included in this meta-analysis, there was no heterogeneity and no publication bias found. The results for this meta-analysis were robust and reliable, and we concluded that $5000 \mathrm{IU}$ of LDUH given twice or three times daily is no more effective than placebo as prophylaxis against VTE for patients with SCI. This conclusion is the same as the current view of this point. ${ }^{4}$ One $\mathrm{RCT}^{25,26}$ compared fixed-dose LDUH (heparin $5000 \mathrm{IU}$, twice daily) with adjusted-dose LDUH (heparin adjusted to prolong the APTT to 1.5 times), the results showed adjusted-dose LDUH was more effective as prophylaxis against VTE, but associated with a higher incidence of bleeding complications than fixed dose. In this study, patients in the adjusted-dose group received of 13,200 $\pm 2200 \mathrm{IU}$ of unfractionated heparin higher than $10,000 \mathrm{IU}$ of unfractionated heparin in the fixed-dose group. It seems increasing the dose of the heparin may increase the effect of thromboprophylaxis, but bleeding complications also significantly increased. Thus, LDUH was not recommended for thromboprophylaxis in patients with SCI.

LMWHs are obtained by various methods of fractionation or depolymerization of polymeric heparin. They have been shown as effective and safe as unfractionated heparin, and have some advantages in terms of pharmacokinetics and convenience of administration. ${ }^{30}$ Although in our systematic review and meta-analysis, the results showed no significant differences for VTE in LDUH versus LMWH, only major bleeding was lower with LMWH. This conclusion was different to the current view of this point. The SCIRE Research Team systematic reviewed VTE prophylaxis in SCI and concluded that $\mathrm{LMWH}$, in particular enoxaparin, was more effective than LDUH at reducing venous thromboembolic events, and the incidence of bleeding complications appears to be less with LMWH. ${ }^{4}$ On the basis of these evidences, the Consortium for Spinal
Cord Medicine Clinical Practice ${ }^{6}$ and the American College of Chest Physicians $^{8}$ recommended LMWH for thromboprophylaxis in patients with SCI. Although we found in SCIRE Research Team's systematic review, ${ }^{4}$ only four trials included for comparing LDUH and LMWH, and the SCIRE Research Team draw conclusion based on level 1 evidence form two RCTs. In one RCT, the incidence of DVT was $63.3 \%$ with LDUH versus $65.5 \%$ with enoxaparin $(P=0.81)$; the incidence of PE was $18.4 \%$ in the LDUH group versus $5.2 \%$ with enoxaparin $(P=0.03)$; the incidence of major bleeding was $5.3 \%$ with LDUH versus $2.6 \%$ with enoxaparin $(P=0.14)$. Only the incidence of PE was significantly different between two groups. We also recalculated the incidence of combined VTE and found no significantly different between two groups $\left(\chi^{2}=0.57, P=0.449\right)$. In the other RCT, the incidence of DVT was $15.8 \%$ (3/19) with LDUH versus $0.0 \%(0 / 16)$ with tinzaparin $\left(\chi^{2}=2.76, P=0.965\right)$; the incidence of PE was $10.5 \%(2 / 19)$ in the LDUH group versus $0.0 \%$ $(0 / 16)$ with tinzaparin $\left(\chi^{2}=1.79, P=0.181\right)$; the incidence of major bleeding was $10.5 \%(2 / 19)$ with LDUH versus $0.0 \%(0 / 16)$ with tinzaparin $\left(\chi^{2}=1.79, P=0.181\right)$. These two RCTs also included in our study. ${ }^{19,27}$ We also included other seven trails for comparing LDUH and LMWH. The increased sample size may make the conclusions more reliable. And, there are some indirect evidences to support our conclusions. Some meta-analysis for other diseases found the same conclusion like ours. In general medical patients, excluding stroke and myocardial infarction, no significant difference was found in efficacy of thromboembolism prophylaxis between LMWH and LDUH, and a significantly reduced risk of bleeding in favor of LMWH was found. ${ }^{31}$ In patients with cancer, no differences were found in DVT, PE and bleeding who receiving perioperative thromboprophylaxis with LMWH versus LDUH. ${ }^{32}$ LMWH treatment appears to be as effective and safe as LDUH for the initial treatment of nonmassive PE. ${ }^{33}$ Although in some diseases, meta-analysis showed the effectiveness of LMWH compared with LDUH. In orthopedic surgery and general surgery patients, the rates of proximal DVT and PE, respectively, are consistently lower under LMWH than under LDUH, whereas slightly smaller rates of distal DVT are observed under LDUH. ${ }^{34}$ In patients with acute coronary syndrome, enoxaparin was associated with superior efficacy as adjunctive AT therapy compared with LDUH. ${ }^{35}$ Our meta-analysis 
increased the sample size compared with previous systematic reviews. But there are two limitations in our meta-analysis for LDUH versus LMWH. The quality of the included studies is relatively poor. Many of them had important methodological limitations. The significant heterogeneity and publication bias were also found. To verify our conclusion, well-designed RCTs are urgently needed.

In summary, our systematic review and meta-analysis showed that in patients with acute SCI, LDUH have no thromboprophylaxis effect compared with placebo or untreated; LMWH can reduce bleeding risk, but cannot prophylaxis thromboembolism compared with LDUH. Because no good quality studies existed in this setting, well-designed RCTs are urgently needed.

\section{DATA ARCHIVING}

There were no data to deposit.

\section{CONFLICT OF INTEREST}

Funding: This research was supported by grant from Natural Science Foundation of China (No. 81271721) and a Project Funded by the Priority Academic Program Development of Jiangsu Higher Education Institutions.

Author contributions: Hong-Lin Chen and Xiao-Dong Wang contributed to the study design, data collection and analysis, and preparation of the manuscript.

1 Merli GJ, Crabbe S, Paluzzi RG, Fritz D. Etiology, incidence, and prevention of deep vein thrombosis in acute spinal cord injury. Arch Phys Med Rehabil 1993; 74 1199-1205.

2 DeVivo MJ, Krause JS, Lammertse DP. Recent trends in mortality and causes of death among persons with spinal cord injury. Arch Phys Med Rehabil 1999; 80: 1411-1419.

3 Geerts WH, Pineo GF, Heit JA, Bergqvist D, Lassen MR, Colwell CW et al. Prevention of venous thromboembolism: the Seventh ACCP Conference on Antithrombotic and Thrombolytic Therapy. Chest 2004; 126 (Suppl), 338S-400S

4 Teasell RW, Hsieh JT, Aubut JA, Eng JJ, Krassioukov A, Tu L. Spinal cord injury rehabilitation evidence Review Research Team. Venous thromboembolism after spinal cord injury. Arch Phys Med Rehabil 2009; 90: 232-245.

5 Merli GJ, Crabbe S, Paluzzi RG, Fritz D. Etiology, incidence, and prevention of deep vein thrombosis in acute spinal cord injury. Arch Phys Med Rehabil 1993; 74 1199-2205.

6 Consortium for spinal cord medicine: prevention of thromboembolism in spinal cord injury: clinical practice guide-lines for spinal cord medicine. Washington, DC, USA Paralyzed Veterans of America 1997.

7 Agarwal NK, Mathur N. Deep vein thrombosis in acute spinal cord injury. Spinal Cord 2009; 47: 769-772.

8 Geerts WH, Heit JA, Clagett GP, Pineo GF, Colwell CW, Anderson FA et al. Prevention of venous thromboembolism. Chest 2001; 119: 132S-175S

9 Thumbikat P, Poonnoose PM, Balasubrahmaniam P, Ravichandran G, McClelland MR. A comparison of heparin/warfarin and enoxaparin thromboprophylaxis in spinal cord injury: the Sheffield experience. Spinal Cord 2002; 40: 416-420.

10 Worley S, Short C, Pike J, Anderson D, Douglas JA, Thompson K. Dalteparin vs low dose unfractionated heparin for prophylaxis against clinically evident venous thromboembolism in acute traumatic spinal cord injury: a retrospective cohort study. J Spinal Cord Med 2008; 31: 379-387.

11 Jadad AR, Moore RA, Carroll D, Jenkinson C, Reynolds DJ, Gavaghan DJ et al. Assessing the quality of reports of randomized clinical trials: is blinding necessary? Control Clin Trials 1996; 17: 1-12.

12 Wells GA, Shea B, O'Connell D, Peterson J, Welch V, Losos M. The Newcastle-Ottawa Scale (NOS) for assessing the quality of non-randomized studies in meta-analysis. Ottawa Health Research Institute. Accessed 30 January 2013. http://www.ohri.ca/ programs/clinical epidemiology/oxford.asp

13 Phillips B, Ball C, Sackett D, Badenoch D, Straus S, Haynes B. Oxford Centre for Evidence-based Medicine, Levels of Evidence (March 2009). Accessed 30 January 2013. http://www.cebm.net/index.aspx?o=1025
14 Marciniak CM, Kaplan J, Welty L, Chen D. Enoxaparin versus tinzaparin for venous thromboembolic prophylaxis during rehabilitation after acute spinal cord injury: a retrospective cohort study comparing safety and efficacy. PM R 2012; 4: 11-17.

15 Arnold JD, Dart BW, Barker DE, Maxwell RA, Burkholder HC, Mejia VA et al. Gold Medal Forum Winner. Unfractionated heparin three times a day versus enoxaparin in the prevention of deep vein thrombosis in trauma patients. Am Surg 2010; 76 . 563-570.

16 Slavik RS, Chan E, Gorman SK, de Lemos J, Chittock D, Simons RK et al. Dalteparin versus enoxaparin for venous thromboembolism prophylaxis in acute spinal cord injury and major orthopedic trauma patients: 'DETECT' trial. J Trauma 2007; 62: 1075-1081.

17 Hebbeler SL, Marciniak CM, Crandall S, Chen D, Nussbaum S, Mendelewski S. Daily vs twice daily enoxaparin in the prevention of venous thromboembolic disorders during rehabilitation following acute spinal cord injury. J Spinal Cord Med 2004; 27 236-240.

18 Chiou-Tan FY, Garza H, Chan KT, Parsons KC, Donovan WH, Robertson CS et al. Comparison of dalteparin and enoxaparin for deep venous thrombosis prophylaxis in patients with spinal cord injury. Am J Phys Med Rehabil 2003; 82: 678-685.

19 Merli Geno, Geerts William, Ginzburg Enrique, Green David, Lambert Richard, Leduc Bernard et al. Prevention of venous thromboembolism in the acute treatment phase after spinal cord injury: a randomized, multicenter trial comparing low-dose heparin plus intermittent pneumatic compression with enoxaparin. J Trauma 2003; 54: 1116-1124.

20 Merli Geno, Geerts William, Ginzburg Enrique, Green David, Lambert Richard, Leduc Bernard et al. Prevention of venous thromboembolism in the rehabilitation phase after spinal cord injury: prophylaxis with low-dose heparin or enoxaparin. J Trauma 2003, 54: 1111-1115.

21 Lohmann U, Gläser E, Braun BE, Bötel U. Prevention of thromboembolism in spinal fractures with spinal cord injuries. Standard heparin versus low-molecular-weight heparin in acute paraplegia. Zentralb/ Chir 2001; 126: 385-390.

22 Spivack SB, Aisen ML. A comparison of low molecular weight heparin and low dose unfractionated heparin prophylaxis in subacute myelopathy. J Spinal Cord Med 1997; 20: 402-405

23 Green D, Twardowski P, Wei R, Rademaker AW. Fatal pulmonary embolism in spinal cord injury. Chest 1994; 105: 853-855.

24 Merli GJ, Crabbe S, Doyle L, Ditunno JF, Herbision GJ. Mechanical plus pharmacological prophylaxis for deep vein thrombosis in acute spinal cord injury. Paraplegia 1992; 30: 558-562.

25 Green D. Prevention of thromboembolism after spinal cord injury. Semin Thromb Hemost 1991; 17: 347-350.

26 Green D, Lee MY, Ito VY, Cohn T, Press J, Filbrandt PR et al. Fixed- vs adjusted-dose heparin in the prophylaxis of thromboembolism in spinal cord injury. JAMA 1988; 260: 1255-1258.

27 Green D, Lee MY, Lim AC, Chmiel JS, Vetter M, Pang T et al. Prevention of thromboembolism after spinal cord injury using low-molecular-weight heparin. Ann Intern Med 1990; 113: 571-574.

28 Merli GJ, Herbison GJ, Ditunno JF, Weitz HH, Henzes JH, Park CH et al. Deep vein thrombosis: prophylaxis in acute spinal cord injured patients. Arch Phys Med Rehabil 1988; 69: 661-664.

29 Frisbie JH, Sasahara AA. Low dose heparin prophylaxis for deep venous thrombosis in acute spinal cord injury patients: a controlled study. Paraplegia 1981; 19: 343-346.

30 Gray E, Mulloy B, Barrowcliffe TW. Heparin and low-molecular-weight heparin. Thromb Haemost 2008; 99: 807-818.

31 Alikhan R, Cohen AT. Heparin for the prevention of venous thromboembolism in general medical patients (excluding stroke and myocardial infarction). Cochrane Database Syst Rev 2010; 17: CD003747.

32 Akl EA, Terrenato I, Barba M, Sperati F, Sempos EV, Muti P et al. Low-molecular-weight heparin vs unfractionated heparin for perioperative thromboprophylaxis in patients with cancer: a systematic review and meta-analysis. Arch Intern Med 2008; 168 : 1261-1269.

33 Quinlan DJ, McQuillan A, Eikelboom JW. Low-molecular-weight heparin compared with intravenous unfractionated heparin for treatment of pulmonary embolism: a meta-analysis of randomized, controlled trials. Ann Intern Med 2004; 140: $175-183$.

34 Koch A, Ziegler S, Breitschwerdt H, Victor N. Low molecular weight heparin and unfractionated heparin in thrombosis prophylaxis: meta-analysis based on original patient data. Thromb Res 2001; 102: 295-309.

35 Murphy SA, Gibson CM, Morrow DA, Van de Werf F, Menown IB, Goodman SG et al. Efficacy and safety of the low-molecular weight heparin enoxaparin compared with unfractionated heparin across the acute coronary syndrome spectrum: a meta-analysis. Eur Heart J 2007; 28: 2077-2086. 